\title{
The Notification Oriented Paradigm Language to Digital Hardware as an intuitive high-level synthesis tool
}

\author{
A Linguagem do Paradigma Orientado a Notificações Para Hardware Digital Como Uma \\ Ferramenta Intuitiva de Síntese em Alto Nível
}

\author{
Ricardo Kerschbaumer ${ }^{1 *}$, André Augusto Kaviatkovski², Gabriel Rodrigues Garcia², Carlos \\ Raimundo Erig Lima², Jean Marcelo Simão²
}

\begin{abstract}
The parallelism allowed by FPGAs has attracted attention for knowing applications that need processing power. However, the need for specific and very technical development language has not stimulate its broad use. As an alternative, there are High-level Synthesis Languages (HSL), which allow less complicated FPGA use. However, they do not tend to take full advantage of the FPGA technology. Therefore, another alternative was developed, based on the Notification Oriented Paradigm (NOP), called NOP for Digital Hardware (NOP-DH). NOP allows development in high level with its rule-oriented language called NOPL. Its entity decoupling, parallelism, and redundancy avoidance are useful for best performance. In turn, the NOP-DH brings NOP for the FPGA context with the benefits observed in software but enhanced by hardware nature. This paper reviews the NOPL for NOP-DH (NOPL-DH) that aims high level programming for FPGA. The paper proposes the NOPL-DH test by independent developers, by developing a monitoring device for a box transporting bidirectional conveyer. As a result, NOPL-DH allowed high-level development under the NOP-DH structure in an FPGA, without the need for technical knowledge and, still, maintaining and exploring the NOP properties in FPGA

Keywords: FPGA, Notification Oriented Paradigm (NOP), Notification Oriented Paradigm to Digital Hardware (NOP-DH), NOP Language (NOPL), NOPL-DH, VHDL
\end{abstract}

Resumo: O paralelismo permitido pelas FPGAs chama a atenção para aplicações que demandam poder de processamento. Porém, a necessidade de linguagem de desenvolvimento muito específica e técnica como VHDL não tem estimulado seu amplo uso. Alternativamente, existem Linguagens de Síntese em Alto Nível que permitem uso menos complicado. No entanto, dado a natureza sequencial delas, elas não tendem a aproveitar todas as vantagens de FPGA como paralelismo. Portanto, foi desenvolvida uma alternativa em Hardware Digital para FPGA, baseada no Paradigma Orientado à Notificação denominado PON-HD. O PON permite o desenvolvimento em alto nível com sua linguagem orientada a regras chamada LingPON, bem como a prevenção de redundância e o correlato desacoplamento de entidades que permitem desempenho apropriado e paralelismo. Por sua vez, o PON-HD traz a solução para as FPGAs com os benefícios observados no software, mas aprimorados pela natureza do hardware. Este artigo revisa a LingPON-HD como programação em alto nível para FPGAs e propõe seu teste por desenvolvedores independentes para um dispositivo de monitoramento de esteira para transporte de caixas. Como resultado, a LingPON-HD permitiu o desenvolvimento em alto nível, via a estrutura PON-HD em FPGA, sem a necessidade de conhecimentos técnicos.

Palavras-Chave: FPGA, Paradigma Orientado a Notificações (PON), Paradigma Orientado a Notificações para Hardware Digital (PON-HD), Linguagem PON (LingPON), LingPON-HD, VHDL

${ }^{1}$ Instituto Federal de Educação Ciência e Tecnologia Catarinense (IFC), Blumenau - Santa Catarina, Brasil

${ }^{2}$ Programa de Pós-graduação em Engenharia Elétrica e Informática Industrial (CPGEI) - Universidade Tecnológica Federal do Paraná (UTFPR), Curitiba - Paraná, Brasil

*Corresponding author: ricardo.kerschbaumer@ifc.edu.br

Este trabalho teve o apoio da Coordenação de Aperfeiçoamento de Pessoal de Nível Superior (CAPES) no doutoramento de Ricardo Kerschbaumer. Este trabalho também teve o apoio da Fundação Araucária (FA) através do Edital CP 15/2017 na bolsa PQ de Jean Marcelo Simão.

DOI: http://dx.doi.org/10.22456/2175-2745.112006 • Received: 09/03/2021 • Accepted: 07/08/2021

CC BY-NC-ND 4.0 - This work is licensed under a Creative Commons Attribution-NonCommercial-NoDerivatives 4.0 International License. 


\section{Introdução}

O aumento da importância da computação em várias áreas cria crescente demanda por mais poder de processamento. Entretanto, arquiteturas de computação tradicionais têm dificuldade em acompanhar este crescimento, principalmente devido a seu modelo de memória e processamento [1] [2]. Assim, a necessidade de novas arquiteturas de computação é cada vez maior e, neste sentido, as Field Programmable Gate Arrays (FPGAs) se mostram como uma alternativa [1] [2] [3].

Entretanto, em suma, o uso de FPGAs apresenta dois problemas. O primeiro problema é que as formas de programação usuais são muito técnicas, o que dificulta o desenvolvimento ainda que permita explorar o inerente paralelismo daquela tecnologia. O segundo problema é que, apesar de ter formas de programação em alto nível facilitadoras do desenvolvimento, elas são oriundas de abordagens sequenciais não permitindo, portanto, explorar com veemência tal paralelismo [4]. Isto posto, este artigo revisa uma solução alternativa que permite programar as FPGAs em alto-nível e implicitamente explorar seu paralelismo de hardware.

A solução em questão se dá à luz de uma nova forma de computação chamada Paradigma Orientado a Notificações (PON), sua linguagem de programação (à priori para software) chamada LingPON e sua tecnologia para hardware digital (HD) chamada de PON-HD, as quais este artigo revisa em português, sendo isto uma primeira contribuição dele [4] [5] [6] [7]. A solução revisada é chamada LingPON para Hardware Digital (LingPON-HD). Ademais e sobretudo, a fim de testar sua usabilidade em alto nível, este artigo propõe um sistema feito com a LingPON-HD por dois desenvolvedores independentes.

O artigo está organizado como segue: a seção 2 apresenta o sistema visado pelos desenvolvedores; a seção 3 revisa os fundamentos do PON e sua Tecnologia LingPON; a seção 4 detalha o PON-HD e sua Tecnologia LingPON-HD; a seção 5 propõe uma solução em LingPON-HD para o sistema visado e apresenta os resultados obtidos; a seção 6 conclui o artigo.

\section{O sistema de monitoramento de esteira}

O Sistema de Monitoramento de Esteira apresentado neste artigo tem servido como elemento de apoio para disciplinas como Microcontroladores, Controle, Lógica Reconfigurável e afins na UTFPR - Campus Curitiba - Departamento Acadêmico de Eletrônica (DAELN). Trata-se de um sistema não complexo que, entretanto, é complicado porque exige a utilização de mais de um tipo de interface, como sensores e atuadores, servindo como um problema com ordem sistêmica condizente para a formação de graduação em engenharias pertinentes [8][9].

Naturalmente, o sistema em questão apresenta-se também como um apropriado ponto de partida para interessados se adaptarem com novas tecnologias em hardware e controle. Neste contexto, o sistema proposto se mostrou adequado ao objetivo de pesquisa sintetizado neste artigo. O sistema pro- posto em si se apresenta com a problemática de monitorar uma esteira bidirecional que transporta caixas de papelão com produtos do setor de embalagem para o setor de carga [8][9].

No sistema dado, de um lado, os produtos são embalados nas caixas e colocados na esteira e, do outro lado, as caixas são coletadas e colocadas em um pallet. Há três tamanhos de caixas: Pequeno (P), Médio (M) e Grande (G). A ordem é aleatória, podendo ser colocado qualquer tipo de caixa na esteira a qualquer momento. Porém, a capacidade de cada pallet é limitada a um arranjo qualquer de caixas $(\mathrm{P}, \mathrm{M}, \mathrm{G})$ [8][9].

A esteira é acionada por um motor de passos que gira continuamente no sentido horário. Ainda, existem três sensores ópticos posicionados assimetricamente ao longo dela, com a função de detectar o tamanho da caixa. O sensor S1 está a uma distância "A" do sensor S2, enquanto o sensor S2 está a uma distância "B" do sensor S3, sendo que se considera B > A, conforme Figura 1 [8][9].

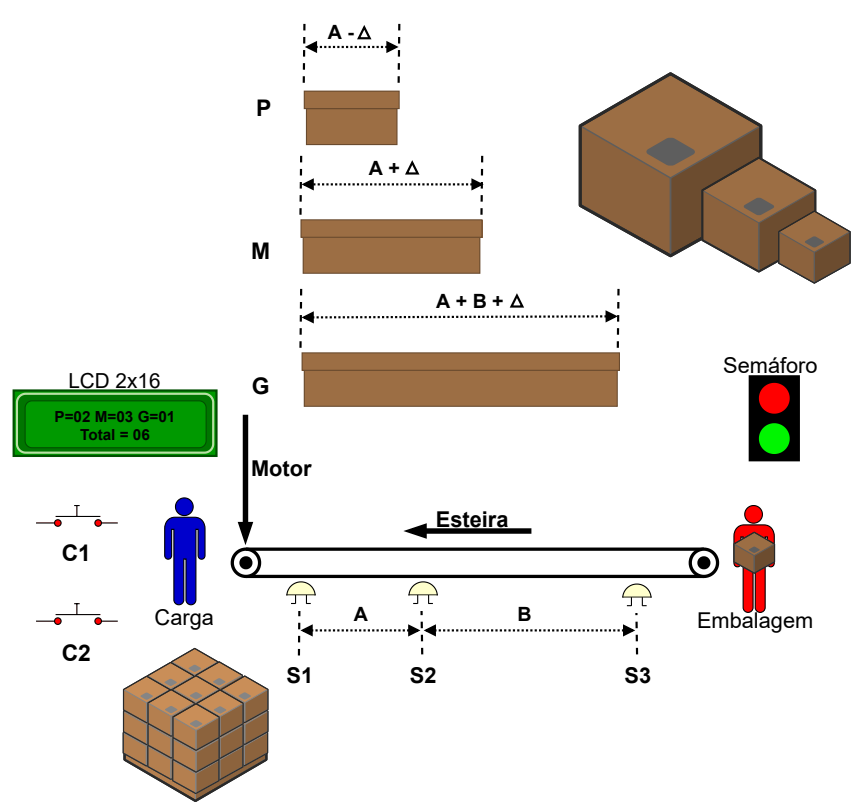

Figura 1. Representação da esteira e seus sensores [9].

Ao lado do setor de carga, há um painel responsável por mostrar continuamente o número de caixas de cada tipo já colocado no pallet, o qual inicialmente é zerado. Há também um semáforo cuja função de sinalizar ao operador se a esteira está disponível ou não para se colocar uma nova caixa [8][9].

O semáforo ficará verde se houver espaço no pallet para a caixa que vai passar pela esteira. Em tempo, na inicialização do sistema, o semáforo também fica verde, pois não há nenhuma caixa na esteira ou no pallet. O semáforo ficará vermelho em duas situações: (a) quando o total de caixas transportadas pela esteira atingir o limite; ou (b) quando o operador do setor de carga notar que não há espaço suficiente no pallet para acomodar a caixa que acaba de passar pela esteira. Neste caso, ele sinaliza via chave $\mathrm{C} 1$ esta situação pressionando uma chave. No caso de chegada de novo pallet com espaço ou afins, ele usará a chave $\mathrm{C} 2$ para sinalizar que há então espaço 
[8][9].

No tocante ao monitoramento, este problema foi resolvido por uma equipe independente, utilizando-se da ferramenta LingPON-HD 1.0 e, consequentemente, a Tecnologia PONHD 1.0. Neste contexto, faz-se necessária e enseja-se à revisão do PON e sua LingPON que constituem a chamada Tecnologia LingPON, bem como do PON-HD e da sua LingPON-HD 1.0 que constituem a assim chamada Tecnologia PON-HD 1.0.

\section{O Paradigma Orientado a Notificações}

Esta seção revisa o Paradigma Orientado a Notificações (PON) resumindo conteúdos apresentados nas referências.

\subsection{Paradigma Orientado a Notificações (PON)}

O PON, como paradigma de desenvolvimento, apresenta um novo conceito para desenvolver e executar sistemas computacionais, com base em entidades-regras, compostas de subentidades colaborativas, as quais são notificáveis a partir de entidades facto-execucionais. A essência do PON é seu processo de inferência baseado em subentidades pequenas e desacopladas, que colaboram entre si por meio de notificações pontuais a fim realizar todo o cálculo lógico-causal [10] [11].

O PON resolve problemas de redundância e centralização das abordagens atuais de processamento lógico-causal, resolvendo assim as questões de mau uso da capacidade de processamento e acoplamento dos paradigmas vigentes [10] [11].

O objetivo do PON é tornar mais fácil a tarefa de criação de sistemas computacionais (e.g., aplicativos e programas), levando-os a serem melhores em termos de facilidade de composição e de código performante e paralelizável / distribuível [12] [13] [14].

\subsection{Inferências das Entidades do PON}

No PON, as expressões causais são representadas por regras lógico-causais usuais, as quais são naturais para os desenvolvedores dos paradigmas atuais de programação. Tecnicamente, cada regra lógico-causal é tratada com uma entidade computacional especial chamada Rule [12] [13]. Uma entidade Rule, relativa a uma regra lógico-causal, é ilustrada na Figura 2 em um formato amigável ou em alto nível [12] [13].

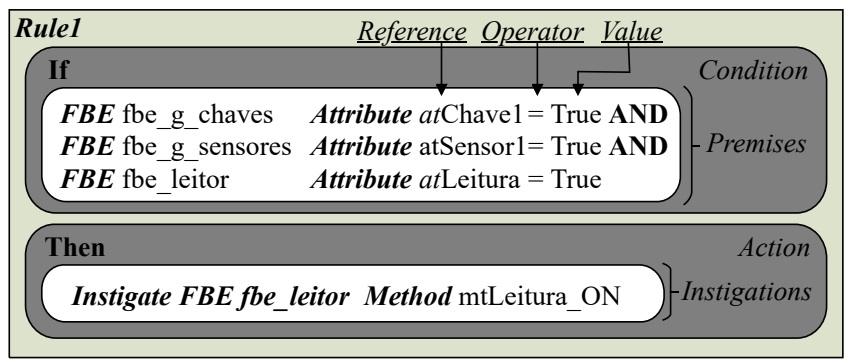

Figura 2. Representação de uma Rule.

Estruturalmente, cada Rule é composta por uma Condition e uma Action, como mostrado por meio do diagrama de blocos em SysML na Figura 3. A Condition e a Action são entidades que trabalham juntas para tratar o conhecimento causal da Rule. Cada Condition é responsável pela tomada de decisão na sua Rule, enquanto cada Action é responsável pela conclusão relativa à uma decisão positiva na sua Rule.

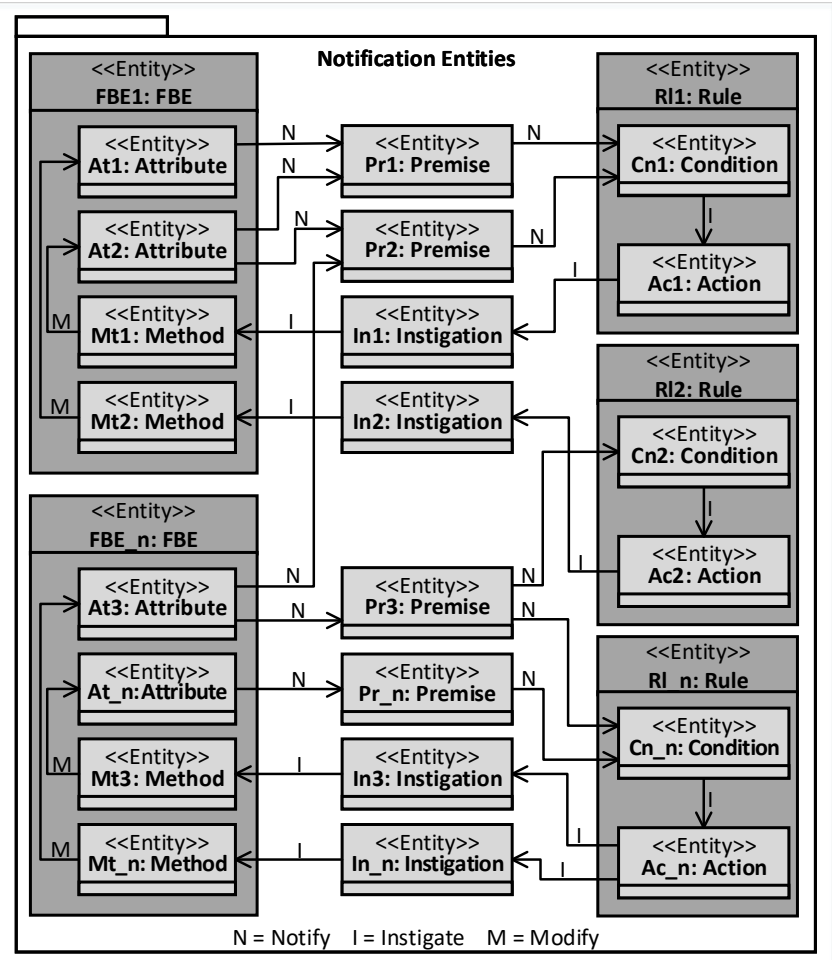

Figura 3. Diagrama de blocos em SysML do ciclo de notificações do PON [9].

Neste âmbito, a Condition avalia estados de elementos factuais, enquanto a Action instiga a execução dos serviços. No exemplo apresentado na Figura 2, estes elementos são o fbe_g_chaves, fbe_g_sensores e fbe_leitor (com o g denotando grupo). Naturalmente, o exemplo já é relativo ao sistema de monitoramento de esteira considerado neste presente artigo.

Cada elemento avaliado no PON por uma ou mais Rules é representado por um tipo de entidade chamado Fact Base Element (FBE). Um FBE é normalmente composto por um conjunto não vazio de atributos. Cada atributo é representado por um outro tipo de entidade chamada Attribute. No exemplo, atChave1, atSensorl e at_Leitura são respectivamente Attributes de fbe_g_chaves, fbe_g_sensores e fbe_leitor.

Os estados dos Attributes são fatos analisáveis em um processo de inferência nas Conditions das Rules. Entretanto, isso se dá por meio de outras entidades chamadas Premises, como apresentado na Figura 3 [12] [13]. Na Rule do exemplo da Figura 2, a Condition é composta por três Premises.

Uma vez que cada Premise de uma Condition é inferida como verdadeira, a Condition torna-se verdade aprovando sua Rule. Uma Rule aprovada pode ativar sua Action, a qual é composta por entidades chamadas Instigations [12] [13].

Na Rule do exemplo, a Action contém uma Instigation. 
Em suma, cada Instigation instiga um conjunto de Methods. Por sua vez, cada Method é outra entidade da FBE, que permite a execução dos serviços desta. Geralmente, a chamada de um Method de uma FBE altera os estados de Attributes de FBE, alimentando assim o processo de inferência [12] [13].

O processo de inferência do PON é diferenciado, uma vez que as Rules têm sua inferência efetuada pela colaboração ativa de suas entidades notificantes, a partir dos FBEs pertinentes [10]. Em resumo, a colaboração acontece da seguinte forma: para cada alteração no estado de um Attribute de uma FBE, a avaliação do estado deste Attribute ocorre apenas nas Premises relacionadas e, então, somente nas Conditions relacionadas e pertinentes, por meio de notificações pontuais entre os colaboradores, tudo no âmbito da chamada Inferência Orientada a Notificações [12] [13].

\subsection{Inferência Orientada a Notificações do PON}

Para explicar o processo de Inferência Orientada a Notificação (ION), primeiramente é necessário explicar a natureza e a composição da Premise. Cada Premise lida com um valor booleano, relacionado com um ou mesmo dois estados de Attributes, sendo composta por: (a) uma referência ao valor de um Attribute recebido por notificação, a qual é chamado de Reference; (b) um operador relacional, o qual é chamado de Operator e é usado para fazer comparações; e (c) outro valor, o qual é chamado de Value e pode ser uma constante ou o valor de outro Attribute recebido também por notificação [12] [13].

Uma Premise faz um cálculo lógico quando recebe uma notificação de um ou de dois Attributes (ou seja, Reference ou Value). Este cálculo é realizado comparando-se a Reference e o Value, usando o Operator. Por sua vez, cada Premise colabora com a avaliação causal de um conjunto de Conditions. Se for alterado o valor booleano de uma Premise notificada por Attribute, então as Conditions relacionadas são notificadas da mudança na Premise por ela própria [12] [13].

Assim, cada Condition notificada calcula seu valor booleano por meio de um operador lógico, na forma de uma conjunção ou uma disjunção, dos valores das Premises pertinentes. No caso de uma conjunção (operador $A N D$ ), por exemplo, quando todas as Premises que integram a Condition são satisfeitas, a Condition em si é satisfeita. No caso de uma disjunção (operador $O R$ ), como outro exemplo, quando uma das Premises que integra a Condition é satisfeita, a Condition em si é satisfeita. Uma vez que a Condition é satisfeita, ela notifica a respectiva Rule para que seja aprovada [12] [13].

Cada Rule aprovada que esteja sem conflito com outra Rule ou tenha esse conflito resolvido pode ser executada. Para executar, a Rule notifica sua Action, a qual a seu turno notifica cada uma das suas Instigations. Por fim, cada Instigation notifica cada um de seus Methods nela referenciados, instigando-os a executar [12] [13]. A colaboração entre as entidades do PON por meio de notificações pode ser observada no diagrama de blocos ilustrado na Figura 3 [9].

Por fim, um ponto importante a explicitar sobre as enti- dades colaborativas do PON é que cada notificador (e.g., um Attribute) leva em conta seus notificáveis (e.g., Premises) no momento da sua criação. Assim, quando uma Premise é criada e faz referência a um Attribute, este último automaticamente leva em conta o anterior no seu conjunto interno de elementos para ser notificado quando seu estado for alterado [12] [13].

\subsection{A natureza do PON}

No PON, cada entidade factual Attribute é avaliado por um conjunto de entidades lógicas e causais, ou seja, as Premises e as Conditions na mudança de seu estado. Graças à cooperação por meio de notificações pontuais entre essas entidades factuais, lógicas e causais, o PON evita os dois tipos de redundâncias verificadas em muitas linguagens usuais de programação dos paradigmas imperativo e mesmo do declarativo. Tais redundâncias geram desperdício de processamento e mesmo acoplamento entre partes do código [12] [13].

Esses dois tipos de redundâncias são a redundância temporal e a redundância estrutural. A redundância temporal consiste na avaliação desnecessária de expressões lógico/causais. Por exemplo, uma expressão dada "se $(\mathrm{A}>500)$ então" não aprovada por valor atual da variável $\mathrm{A}$, sendo reavaliada outra(s) vez(es) em um laço de repetição sem ter havido mudanças na variável A. A redundância estrutural, por sua vez, é a repetição de expressão lógica no âmbito de expressões causais [12] [13]. Por exemplo, uma expressão lógica dada $(\mathrm{A}>500)$ repetida no âmbito de duas ou mais expressões "se então".

A redundância temporal é resolvida no PON eliminando a pesquisas sobre elementos passivos. Em primeiro lugar, os Attributes são reativos em relação a atualização de seu estado. Em segundo lugar, os Attributes notificam pontualmente apenas as Premises que estão interessadas na atualização de seu estado. Por sua vez, Premises notificam pontualmente apenas as Conditions pertinentes. Portanto, evita-se que expressões lógico-causais sejam reavaliadas desnecessariamente [12] [13].

A redundância estrutural é também resolvida no PON. Isso acontece quando uma Premise é compartilhada por duas ou mais Conditions. Assim, a Premise realiza o cálculo lógico apenas uma vez e compartilha o resultado lógico apenas com as Conditions relacionadas, evitando assim reavaliações desnecessárias e a repetição de Premises iguais [13].

Desta forma, evitando redundâncias estruturais e, principalmente, evitando redundâncias temporais, é possível obter melhora no desempenho [13]. Além de auxiliar a resolver problemas de desempenho, o PON é também potencialmente aplicável no desenvolvimento de aplicações paralelas ou distribuídas. Isto ocorre devido ao "desacoplamento" de suas entidades (ou acoplamento mínimo entre entidades para ser mais preciso) permitido pela orientação a notificações [14].

Em termos de inferência, não há diferença fundamental se uma entidade é notificada na mesma região de memória, na memória do mesmo computador ou na mesma sub rede. Por exemplo, uma entidade notificante (e.g., um Attribute) pode 
estar em execução em uma máquina ou processador e a entidade notificável (e.g., uma Premise) pode estar em execução em outro. Para o notificador, é necessário 'somente' saber o endereço da entidade notificável [12] [13]. Entretanto, obviamente, conforme a natureza do ambiente, como ambientes assíncronos, pode-se ser necessário mecanismo suplementar caso se almeje determinismo e/ou resolução de conflitos [15].

\subsection{As plataformas do PON}

O PON já foi implementado em plataformas diferentes para apoiar o desenvolvimento e execução de aplicativos nele. A primeira implementação foi um arquétipo ou framework $\mathrm{C}++$, com quatro diferentes versões. Estas versões foram ainda derivadas para lidar com diferentes situações, como aplicações multi-thread, plataformas multi-core, sistemas fuzzy e redes neurais. Além disso, versões do framework foram reconstruídas em outras linguagens de programação, como Java e C\#. Assim, nas aplicações PON desenvolvidas nestes frameworks, os objetos atuam como entidades notificantes [5] [14] [16].

Estes frameworks permitiram demonstrar a viabilidade do PON e suas propriedades, como programação em alto nível, a facilidade da distribuição do código e o desempenho adequado. No entanto, pelo fato de se tratar de frameworks sobre outras linguagens de programação, o desempenho não era tão efetivo como previsto em teoria e a facilidade de implementação em alto nível poderia também ser melhorada. Assim, foi elaborada uma linguagem de programação e seu respectivo compilador, chamado de Tecnologia LingPON, a luz de método próprio para criação de linguagens e compiladores para o PON [7] [16].

A tecnologia LingPON permite gerar código em framework $\mathrm{PON} \mathrm{C}++$, código específico notificante em $\mathrm{C}$ ou $\mathrm{C}++$, entre outros, tudo a partir de uma linguagem de alto nível. Em suma, o nível de abstração de desenvolvimento e o desempenho foram melhorados. Ainda, a Tecnologia LingPON está em evolução buscando melhorias diversas, já havendo versões com implementações multi-threaded. Existe tal qual adaptações da tecnologia para sistemas nebulosos (ou fuzzy) e redes neurais, bem como para os esforços do PON em hardware [5] [6] [16].

Ainda, houve outros esforços para desenvolver uma arquitetura na qual o PON é implementado parte em hardware e parte em software. Neste esforço, a parte implementada em hardware é um coprocessador (CoPON) responsável pelas avaliações lógico-causais. A parte em software é um programa que lida com o processamento factual e execucional em núcleo Von Neumann comum. Uma implementação desta arquitetura foi feita em lógica reconfigurável (FPGA) e significativamente reduziu o número de ciclos de clock necessários para executar as tarefas quando comparado com Framework PON C++ [17].

Além disso, foi desenvolvida uma arquitetura de computação para o PON, a ArqPON (ou, em inglês, Notification Oriented Computing Architecture - NOCA). Ela foi usada como base para implementar um microprocessador PON completo em uma FPGA. Esta implementação foi capaz de buscar em memória e executar aplicações PON desenvolvidas como um software de baixo nível, busca essa de forma semelhante a um processador Von Neumann comum, porém com uma dinâmica de execução via propagação de notificações, o que é mais próximo à dinâmica definida pelo modelo teórico do PON [18] [19].

Por fim, para tratar aplicações realmente paralelas, uma implementação do PON puramente no hardware digital (PONHD) foi desenvolvida [15]. Nesta solução, o desenvolvedor pode usar a expressividade do paradigma para o projeto da aplicação aproveitando, ademais, o paralelismo de execução das FPGAs. Esta solução foi objeto de um pedido de patente em 2012 [11]. Subsequentemente, houve evolução substancial na abordagem do PON-HD, a qual é usada nos experimentos apresentados neste artigo. A próxima seção apresenta o PONHD em detalhes.

\section{O PON em Hardware Digital}

A implementação do PON-HD utilizada neste trabalho é uma interpretação do pedido de patente pertinente, bem como uma veemente evolução de respectiva solução precedente [11] [12]. Isto posto, esta seção resume os conteúdos de PON-HD apresentados nas seguintes referências [20] [21]. Entretanto, o exemplo apresentado aqui é certamente pertinente ao sistema de monitoramento de esteira visado neste presente artigo.

\subsection{A implementação do PON-HD}

$\mathrm{Na}$ implementação do PON em Hardware Digital (HD), os elementos que constituem este paradigma foram implementados como componentes VHDL, formando assim um framework. Em tempo, alguns dos componentes do PON servem para organizar e agrupar outros elementos e não possuem correspondentes físicos, portanto não integrando o framework PON-HD, que é chamado apenas de PON-HD.

A Figura 4 reapresenta o diagrama de blocos da Figura 3 , mas com os elementos do PON que não possuem correspondentes físicos no PON-HD marcados como $<<$ Conceptual $>>$. Um exemplo é o FBE, que serve para agrupar Methods e Attributes e não tem (por hora) função no hardware gerado.

A operação do PON-HD pode ser resumida em: (a) os valores dos Attributes são comparados pelas Premises, resultando em um valor lógico (verdadeiro ou falso); (b) se a operação lógica definida pela Condition, sobre as Premises que a compõem, apresentar um resultado verdadeiro, os respectivos Methods são ativados; (c) os Methods ativados alteram o valor de outro Attribute, perfazendo o ciclo de execução no PON-HD [9].

Um pequeno exemplo de uma aplicação em PON-HD é apresentado na Figura 5. O circuito deste exemplo é relativo a Rule apresentada no formato de regra se-então em alto nível na Figura 2.

Nesta Figura 5, considera-se o seguinte: 


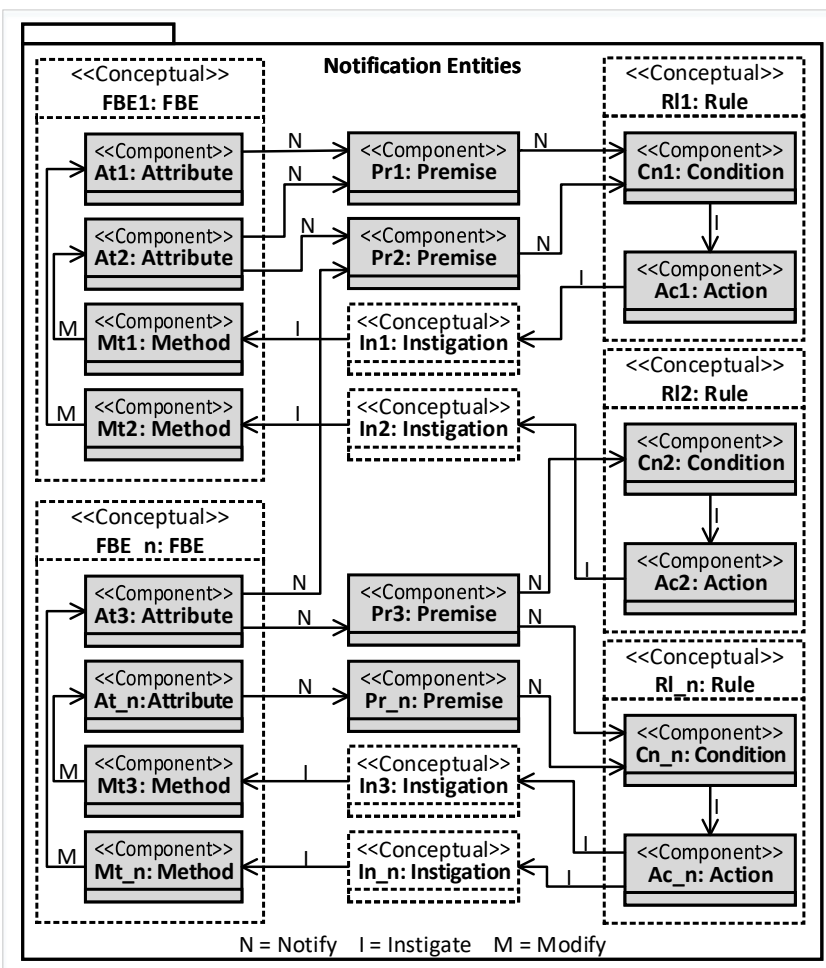

Figura 4. Diagrama de blocos em SysML do ciclo de notificações do PON.

- A primeira Premise é chamada "prChave1_ON_EQUAL" e verifica se o Attribute "atChave1" é verdadeiro. Suas entradas são: o valor do Attribute "atChave1" e a constante 1 .

- A segunda Premise é chamada "prLeitura_ON_EQUAL" e verifica se o valor do Attribute "atLeitura" é verdadeiro. As entradas são: o valor do Attribute "atLeitura" e a constante 1.

- A terceira Premise é chamada "prSensor1_ON_EQUAL" e verifica se o valor do Attribute "atSensor1" é verdadeiro. As entradas são: o valor do Attribute "atSensor1" e a constante 1.

- A quarta Premise é chamada "prSensor1_OFF_EQUAL" e verifica se o valor do Attribute "atSensor1" é verdadeiro. As entradas são: o valor do Attribute "atSensor1" e a constante 1.

- A quinta Premise é chamada "prLeitura_OFF_EQUAL" e verifica se o valor do Attribute "atLeitura" é verdadeiro. As entradas são: o valor do Attribute "atLeitura" e a constante 1.

Há também dois Methods, um chamado "mtLeitura_OFF", cuja função é incrementar o valor do Attribute "atLeitura". As suas entradas são duas constantes de valor 1. O segundo Method é chamado "mtLeitura_ON" cuja função é incrementar o valor do Attribute "atLeitura". As suas entradas são duas constantes de valor 1 .

As entradas do circuito são: o sinal de clock, a entrada "atChave1" que habilita o funcionamento do circuito, a entrada "atSensor1" que habilita a contagem, a entrada "Set_atChave1" que armazena o valor da entrada no Attribute "atChave1" e a entrada "Set_atSensor1" que armazena o valor da entrada no Attribute "atSensor1". A saída do circuito é o valor ar-

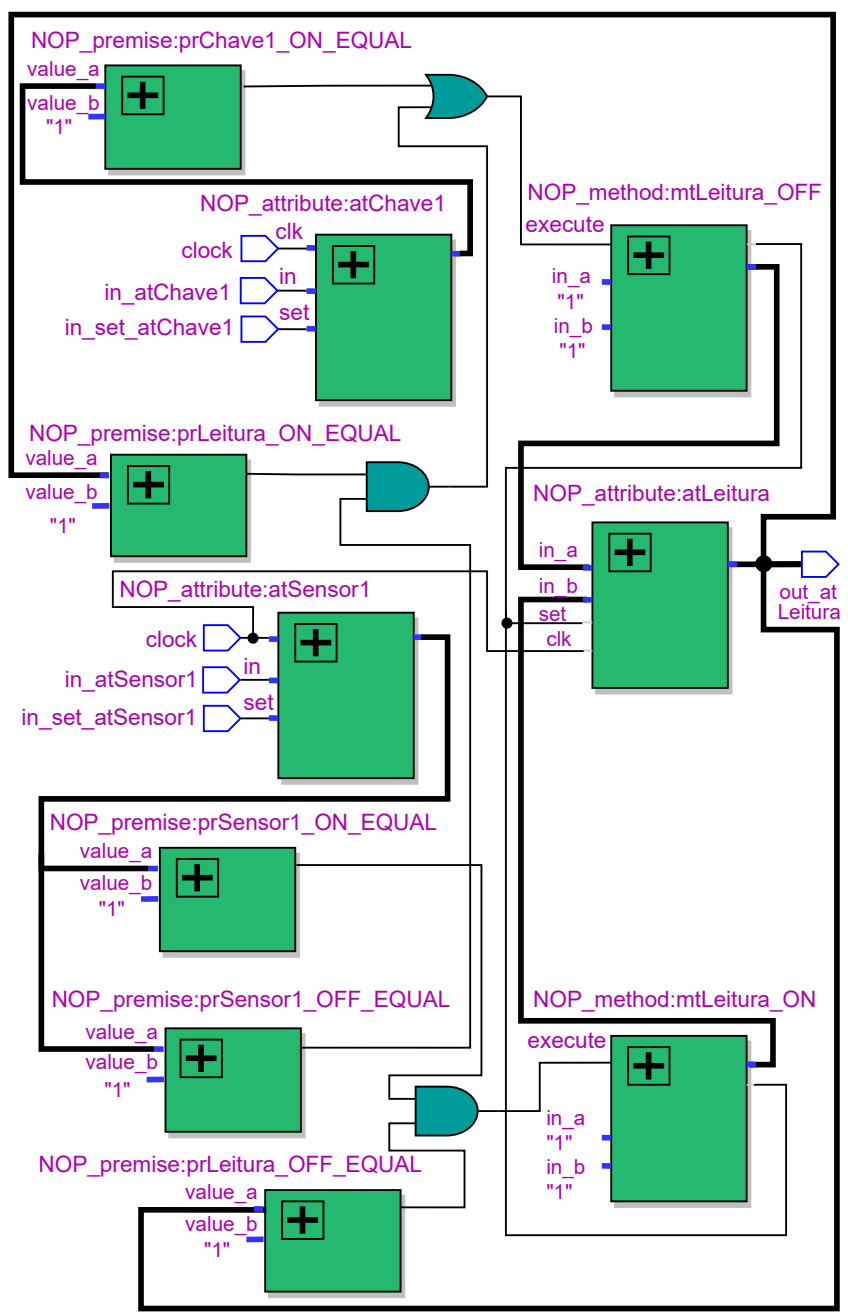

Figura 5. Diagrama esquemático da implementação em hardware.

mazenado no Attribute "atLeitura", que é composto de 1 bit.

A operação do circuito é simples pois, se um dado sinal de ativação é escrito nos Attributes "atChave1" e "atSensor1", então as duas Premises pertinentes, "prChave1_ON_EQUAL" e "prSensor1_ON_EQUAL", tornam-se verdadeiras e ativam o Method "mtLeitura_ON". Esse método incrementa o valor do Attribute "atLeitura" de 0 para 1. Então, as Premises "prSensor1_OFF_EQUAL" e "prLLeitura_OFF_EQUAL" tornam-se falsas e a Premise "prLLeitura_ON_EQUAL" verdadeira, inibindo assim o Method "mtLeitura_OFF" e parando a leitura do sensor.

\subsection{Os componentes do PON-HD}

Para melhor compreensão do funcionamento do PON-HD, é detalhado a seguir o funcionamento de seus componentes no hardware. Também são apresentados detalhes sobre a implementação deles, iniciando pelo Attribute [9] [20].

Um Attribute é responsável pelo armazenamento dos dados no PON, portanto, é natural que no hardware o Attribute seja composto por registradores. O Attribute é o único ele- 
mento sincronizado pelo clock no PON-HD. Assim, todos os outros elementos são totalmente combinacionais. Esta estrutura permite que todo o ciclo de notificação do PON-HD ocorra em um único ciclo de clock [9] [20].

Como o Attribute é implementado na forma de um componente VHDL, o número de bits que ele pode armazenar e o número de entradas que suporta são configuráveis por meio de parâmetros do tipo "generics". O conteúdo armazenado no Attribute é usado pelas Premises e pelos Methods em suas computações [9] [20]. A Figura 6 também apresenta a estrutura de um Attribute de 1 bit com duas entradas.

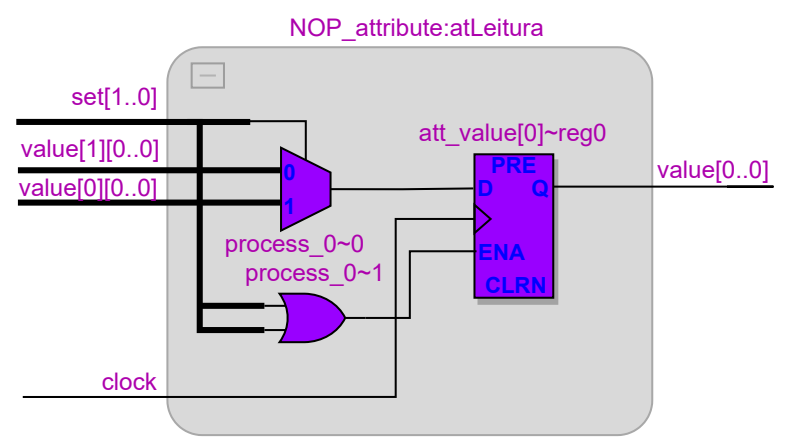

Figura 6. Exemplo de Attribute [12].

Os Methods, por sua vez, são capazes de atualizar o conteúdo dos Attributes, armazenando o resultado de suas computações neles e alimentando assim o ciclo de inferência. Como dois ou mais Methods podem acessar um determinado Attribute ao mesmo tempo, foi implementado um mecanismo de prioridade. Neste, uma entrada de menor índice tem prioridade sobre uma entrada de maior índice. Na Figura 6, a entrada value[0] tem prioridade sobre a entrada value[1] e assim por diante [9] [20].

Os Methods são responsáveis pelas computações usuais no PON-HD. Qualquer circuito combinacional com duas entradas e uma saída pode ser implementado em um Method. Nesta versão, são implementadas apenas operações aritméticas e lógicas simples. Cada Method recebe duas entradas, que podem ser valores constantes ou valores de Attributes [9] [20].

A Figura 7 mostra a estrutura de um Method que executa a operação de soma de valores de 32 bits. Tal qual os Attributes, os Methods são implementados como componentes VHDL. O número de bits das entradas e a operação a ser executada também são configuráveis por meio de "generics" [9] [20].

As Rules, assim como as FBEs, têm a função de agrupar os elementos e, por hora, não têm nenhuma implementação em hardware. Do ponto de vista de PON-HD, cada Rule serve principalmente como uma abstração de alto nível que ajuda a organizar o projeto. Por sua vez, Condition e Action da Rule sim tem papel efetivo no PON-HD vigente [9] [20].

No PON-HD, cada Condition, é responsável por executar uma operação lógica (E ou OU) entre os valores das Premises. Este componente é facilmente implementado em hardware por meio de portas E ou OU. Assim, não é necessário criar um componente VHDL específico para tal. A porta E na Figura 8

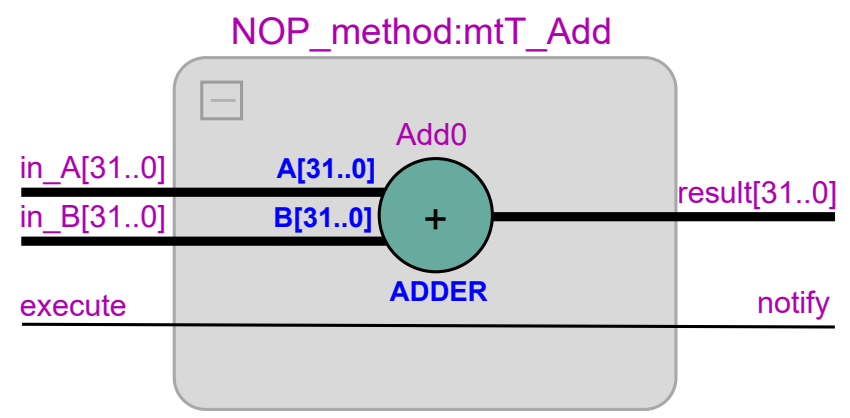

Figura 7. Exemplo de Method [12].

é um exemplo de implementação de uma Condition [9] [20].

As Premises, em tempo, são os elementos responsáveis pelas operações lógico-relacionais no PON-HD. As operações implementadas são: igual, diferente, maior, maior ou igual, menor e menor ou igual. Ainda, uma Premise recebe duas entradas, que podem ser Attributes ou valores constantes. $\mathrm{O}$ resultado da Premise é um valor binário que indica se o condicional de teste foi satisfeito ou não.

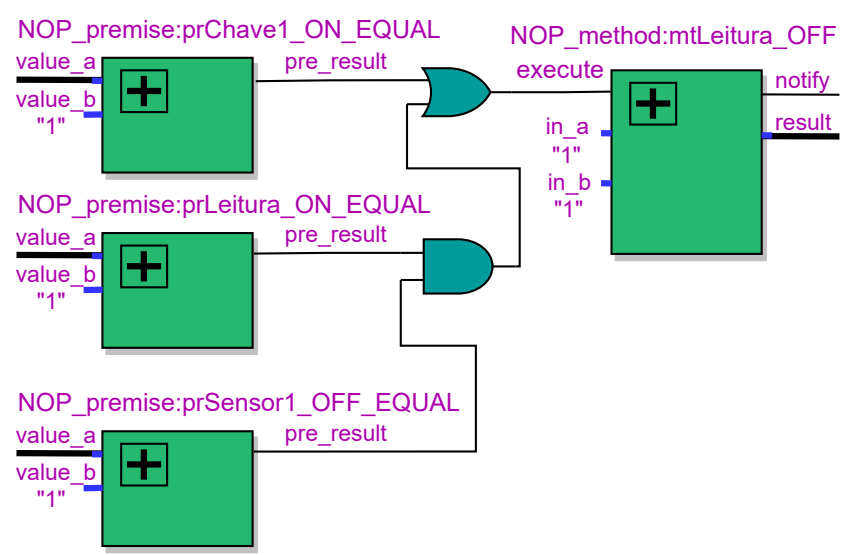

Figura 8. Exemplo de Condition e Action [12].

A Figura 9 mostra a estrutura de uma Premise que executa a operação "diferente" entre valores de 1 bit. A Premise também é implementada como um componente VHDL, o qual permite que o número de bits das entradas e a operação sejam configurados por meio de "generics" [12].

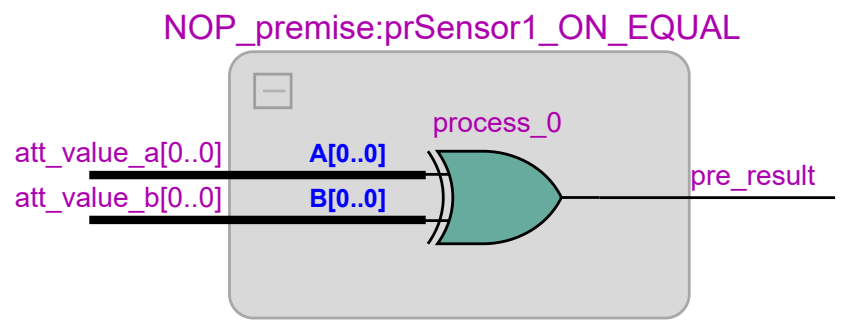

Figura 9. Exemplo de Premise [12].

Por fim, as Actions com as Instigations são responsáveis por transportar o sinal de ativação das Conditions para os 
Methods, seguindo as ligações estabelecidas no projeto. Assim, uma porta OU é suficiente para realizar essa tarefa no hardware, como mostrado na Figura 8. Neste exemplo o Method "mtLeitura_OFF" é ativado quando a Premise "prChave1_ON_EQUAL" for verdadeira ou quando as Premises "prLeitura_ON_EQUAL" e "prSensor1_OFF_EQUAL” forem verdadeiras.

A implementação atual do PON-HD suporta operações com número inteiro e booleanos. No entanto, é fácil adicionar suporte para outros tipos de dados, alterando o código VHDL das Premises e dos Methods. Por exemplo, para adicionar suporte a ponto flutuante, é necessário alterar o código da Premise de forma que seja possível realizar comparações entre números de ponto flutuante. Também é necessário alterar o código dos Methods para executar operações sobre este tipo de dados. Os Attributes não precisam ser alterados, pois eles apenas armazenam dados, independentemente de seu tipo [12].

É possível utilizar o PON-HD para criar hardware digital de duas formas distintas. Os componentes do PON-HD podem ser instanciados manualmente em um arquivo VHDL, no qual o desenvolvedor é responsável por fazer todas as conexões e parametrizações. Outra forma de utilizar o PONHD é por meio de um compilador. Neste caso o desenvolvedor cria a aplicação em linguagem de alto nível, a LingPON, e o compilador automaticamente gera o arquivo VHDL com as conexões e parametrizações necessárias [12]. De qualquer forma, o código VHDL resultante pode ser compilado, simulado e transferido para um FPGA com as ferramentas do fabricante do hardware.

\subsection{Linguagem e compilador PON}

O PON como solução de desenvolvimento carecia de melhores facilidades de programação. Isto porque mesmo com o uso de frameworks as aplicações em PON não permitiam uma perfeita visualização de seus componentes na forma orientada a regras em altíssimo nível no código. Assim, não tinha o mesmo apelo e organização que uma linguagem específica permitiria.

Portanto, foi desenvolvida uma linguagem de programação específica do PON e um compilador, capaz de traduzir tal linguagem em código-alvo, a luz de método próprio para construção de linguagens e compiladores para o PON. A linguagem/compilador inicial desenvolvida foi chamada de Tecnologia LingPON 1.0. Na LingPON se escreve aplicações orientada a regras em alto nível. Ainda, o compilador permite traduzir este código orientado a regras para código notificante em linguagens $\mathrm{C}$ e $\mathrm{C}++$ (portanto provendo-as de nova conotação), em Framework PON C++ 2.0, entre outros [7] [16].

A Tecnologia LingPON primeiramente foi desenvolvida para criar aplicações de software em PON para computadores usuais (i.e., von Neumann), não apresentado todas as características necessárias a descrição de hardware digital. Assim, fez-se necessário criar uma derivação dela com as características necessárias a descrição de hardware digital [9] [21].

Esta derivação foi chamada de Tecnologia LingPON-HD. Ela acrescenta à LingPON alguns elementos necessários à geração de código para hardware digital. A compatibilidade entre as duas variantes da linguagem foi mantida, bastando apenas acrescentar alguns comandos a um programa em LingPON para torná-lo compatível com a LingPON-HD [9] [21]. O compilador em si também teve que ser alterado de forma a permitir obter código em lógica reconfigurável a partir da tradução do código LingPON-HD para PON-HD em VHDL, possibilitando assim sua execução diretamente em hardware [9] [21].

\subsection{LingPON-HD - uma ferramenta de síntese de alto nível}

O PON-HD em si já fornece ao desenvolvedor abordagem de mais alto nível para o problema da síntese de hardware. $\mathrm{O}$ desenvolvedor se preocupa apenas com o fluxo de notificações entre os componentes ou elementos do PON-HD e não com a complexa sintaxe das linguagens de descrição de hardware, dado que os elementos do PON-HD são predefinidos e testados, o que ainda diminuiria a probabilidade de erros [9] [21].

A estrutura do projeto em PON-HD permite que o desenvolvedor se preocupe apenas com um elemento de cada vez. Isto é possível porque os elementos do PON-HD são consideravelmente desacoplados uns dos outros, sendo interligados apenas pelas notificações. Contudo, a principal característica do PON-HD, relacionada a síntese de hardware em alto nível, é a Tecnologia LingPON-HD. Esta permite que todo o código PON-HD da aplicação, em VHDL, seja gerado automaticamente a partir da LingPON-HD. Esta é considerada fácil de aprender já que o desenvolvedor usa um conjunto pequeno de elementos e de sintaxe simples [9] [21].

Ainda, a implementação atual da Tecnologia LingPONHD é interessante para resolver alguns tipos de problemas, mas não é adequado para todos os tipos. Devido à estrutura dela, a manipulação de fluxos de dados, como em filtros digitais ou de processamento de imagens é possível, mas complicada. Como não estão implementados Attributes na forma de vetores, a manipulação deste tipo de dados é dificultosa. Ademais, o PON-HD é mais adequado para problemas de controle orientado a eventos discreto, nos quais a mudança de estado de um elemento exige a tomada de decisões e a alteração do estado de outros elementos via alguma ação [9] [21].

\subsection{Trabalhos Prévios em PON-HD e Tecnologia PON- HD}

Previamente, um conjunto trabalhos demonstraram a utilidade e pertinência de PON-HD em si e também no âmbito da Tecnologia LingPON-HD por meio de experimentos e mesmo bechmarks. Assim, esta seção se dedica a reportar os trabalhos prévios já realizados com PON-HD puro ou com Tecnologia 
LingPON-HD, bem como os seus respectivos resultados, sempre à luz do discutido em [22].

No artigo de Pordeus et al [20], apresentou-se um experimento que consiste na implementação de um simulador telefônico. Os resultados demonstram que a implementação em PON-HD tem performance equivalente e ainda melhor expressividade em termos de nível de abstração que a abordagem equivalente usando VHDL padrão ou via máquina de estados [20]. Ainda no artigo de Pordeus et al. [20] e ademais no artigo de Kerschbaumer et al. [4], apresentaram-se experimentos que consistem em diferentes implementações do algoritmo Bubble Sort com VHDL tradicional e diferentes abordagens de componentes em PON-HD. Estes experimentos demonstraram que o PON-HD permite circuitos com performance e paralelismo apropriados, tendo equivalente quantidade de uso de elementos lógicos e, por vezes, melhor frequência que em VHDL tradicional. Além do mais, PON-HD permite alcançar isto com desenvolvimento em considerável alto nível no seu framework de componentes. Em tempo, nos experimentos desses artigos, ainda não se utilizava a Tecnologia LingPON-HD, a qual é ainda em mais alto nível [4][20].

No artigo de Kerschbaumer et al. [21], apresenta-se um experimento que consiste do controle de um robô hexápode em um ambiente de simulação, nomeadamente o V-REP. Este experimento objetivou avaliar funcionalmente a Tecnologia LingPON-HD, como uma ferramenta de síntese de alto nível em um contexto com decisões mais significativa de controle. Quanto ao robô, ele tem dois sensores ópticos capazes de identificar uma linha no solo a ser seguida, bem como tem seis pernas que permitem sua movimentação. Cada perna tem três motores, totalizando dezoito motores a serem controlados. Como resultado, o robô simulado com o controle em Tecnologia LingPON-HD teve as mesmas decisões e seguiu o mesmo caminho que o mesmo robô simulado com abordagem de controle tradicional em VHDL. Entretanto, em Tecnologia LingPON-HD houve a vantagem de programação declarativa em alto nível [21].

Em trabalhos de Schütz [23] apresentou-se uma solução alternativa de Rede Neural Artificial (RNA) via Tecnologia LingPON, chamada de NeuroPON [24]. A NeuroPON foi usada para RNA do tipo Multilayer Perceptron para funções $X O R$ e Iris Dataset, ambas com treinamento pelo método de backpropagation. Em termos de hardware, a NeuroPON-HD foi elaborada via Tecnologia LingPON-HD [23]. Nos experimentos, considerou-se o número de elementos lógicos e a frequência máxima em relação à VHDL tradicional para abordagem usual de RNA. Os resultados indicam que a abordagem tradicional em VHDL usou um pouco menos de elementos lógicos e teve maior frequência de operação que com a prototipal NeuroPON-HD. Entretanto, a Tecnologia PON-HD permitiu RNA em uma forma declarativa, a NeuroPON. Ainda, a NeuroPON permite customizar cada neurônio em uma cadeia sináptico notificante, o que deverá ser explorado no futuro. Por fim, o NeuroPON permite gerar solução em diferentes plataformas a partir de uma mesma abordagem declarativa

\section{[23].}

Finalmente, no artigo de Pordeus et al. [22], apresenta-se um experimento que avalia o uso de PON-HD para o desenvolvimento do conhecido algoritmo Bitonic Sort como um benchmark. Este algoritmo tem propriedades particulares que são vantajosas para a execução em paralelo, especialmente em FPGAs. O experimento compara a performance, a quantidade de elementos lógicos e frequência máxima do PON-HD em relação a abordagem tradicional de VHDL. Como resultado, estes experimentos demonstraram que o circuito em PON-HD puro alcança resultados similares quando comparado com a abordagem tradicional de desenvolvimento. Ainda, em Tecnologia LingPON-HD, os resultados com código PON-HD gerado são divididos por dois, significando apenas que o compilador naturalmente precisa passar por otimização [22].

Estes experimentos prévios têm demonstrado a viabilidade de PON-HD e Tecnologia LingPON-HD como uma ferramenta de síntese em alto nível, validando pertinência da continuidade de pesquisas nesta abordagem. Entretanto, faltava ainda um teste com desenvolvedores que não conhecessem o PON-HD em detalhes (o que inclui não conhecer o VHDL gerado) e que fizesse uso de Tecnologia LingPONHD em alto nível, apenas da sua linguagem declarativa, para se perceber a viabilidade dela como um uma ferramenta para desenvolvedores não experimentados.

\section{Experimento realizado}

Para demonstrar um primeiro uso da Tecnologia LingPONHD por desenvolvedores não experimentados, enquanto uma ferramenta de síntese em alto nível, foi realizado um experimento, o qual será descrito a seguir. $\mathrm{O}$ experimento consiste em um monitor de quantidades de caixas de papelão, a luz do problema descrito na segunda seção deste presente artigo. Dependendo do tamanho da caixa que passa pela esteira, o monitor informa ao usuário se a caixa é do tamanho grande, médio ou pequeno, conforme já previamente descrito. A quantidade total de caixas que passaram pela esteira é também informada.

Para simular o sistema foi projetado um circuito físico com sensores opto eletrônicos que têm como finalidade gerar o sinal interpretado pelo monitor. Em tempo, este monitor foi elaborado em Tecnologia LingPON-HD, a qual gera código VHDL em PON-HD. Ainda, o monitor informa ao usuário, via displays de sete segmentos, o tamanho e quantidade das caixas. O hardware usado foi a placa DE1 Terasic que utiliza o FPGA Cyclone II da Intel/Altera. A Figura 10 apresenta o hardware utilizado no experimento que simula o sistema controlado.

\subsection{Desenvolvimento do Simulador}

O kit utilizado no projeto com a placa DE1 Terasic foi o DE1 (Development and Educational Bord) da Altera. Foi utilizado alguns dos barramentos da placa para poder representar o problema dado. Os botões KEY 1 e KEY 2 foram usados para representarem o papel das chaves $\mathrm{C} 1$ e $\mathrm{C} 2$ e os leds LEDG 7 


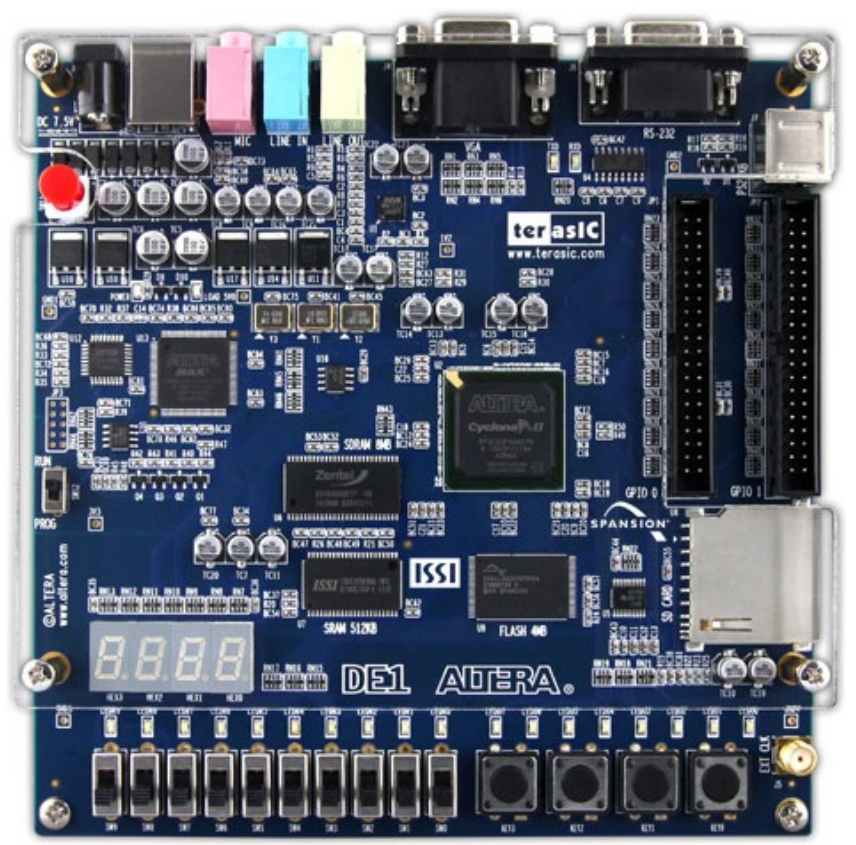

Figura 10. Hardware utilizado no experimento [25].

e LEDR 0 para fazerem o papel do semáforo. Foi utilizado também os leds LEDR 7 e LEDG 8 que representam quando o motor está no sentido horário e anti-horário, respectivamente.

Os sensores utilizados foram do modelo PHCT203 e foram ligados nas portas GPIO do kit. A porta utilizada foi a porta 0 e os pinos utilizados foram GPIO0[0], GPIO0[1] e GPIO0[2]. O motor foi ligado na porta 1 do GPIO, os pinos utilizados foram GPIO1[0], GPIO1[1], GPIO1[2] e GPIO1[3]. Foi utilizado também um switch para definir um reset no motor, precisamente o SW 8, e ainda um outro switch para controlar e acionar todo circuito principal, precisamente o SW 9.

O projeto foi todo modelado no Quartus II em diagramas de blocos. Porém, naturalmente, para a criação do bloco principal, que é justamente o 'monitor de quantidades de caixas de papelão', foi utilizada a tecnologia LingPON-HD para desenvolver a sua lógica, utilizando-se de arquivos com extensão .pon de sua linguagem declarativa. Por meio do compilador da Tecnologia LingPON-HD gerou-se automaticamente um arquivo em VHDL, que posteriormente foi usado no sistema para gerar o bloco do monitor.

Foi necessário também criar um debounce para controlar as entradas das chaves uma vez que as mesmas geravam um ruído muito grande prejudicando o funcionamento correto do projeto. O debounce também foi criado por meio da linguagem da Tecnologia LingPON-HD [3]. O bloco gerado no projeto recebeu o nome de "AntiBounce".

Ainda, o motor de passo foi controlado por meio de uma máquina de estados usual, que já havia sido criado anteriormente sendo este código reutilizado. Por fim, para controlar o display de sete segmentos foi utilizado um código em VHDL encontrado na literatura técnica usual.

\subsection{LingPON-HD - uma ferramenta de alto nível}

No experimento, a lógica de monitoramento dos sensores das entradas e monitoramento das caixas por tamanho, bem como ações daí decorrentes foram feitas em tecnologia LingPONHD por dois desenvolvedores independentes: $2^{\circ}$ e $3^{\circ}$ autores deste artigo.

A seguir é apresentado o programa com os Attributes e Methods, enquadrados em FBEs, relativos ao monitoramento e ações do sistema. Neste código apresentado, para melhorar a sua compreensão, usou-se FBEs relativos aos 14 Attributes e 26 Methods. Também há todas as Rules que constituem parte deste módulo de monitoração feito em Tecnologia LingPONHD. Usou-se 22 Premises relacionadas com 36 Rules.

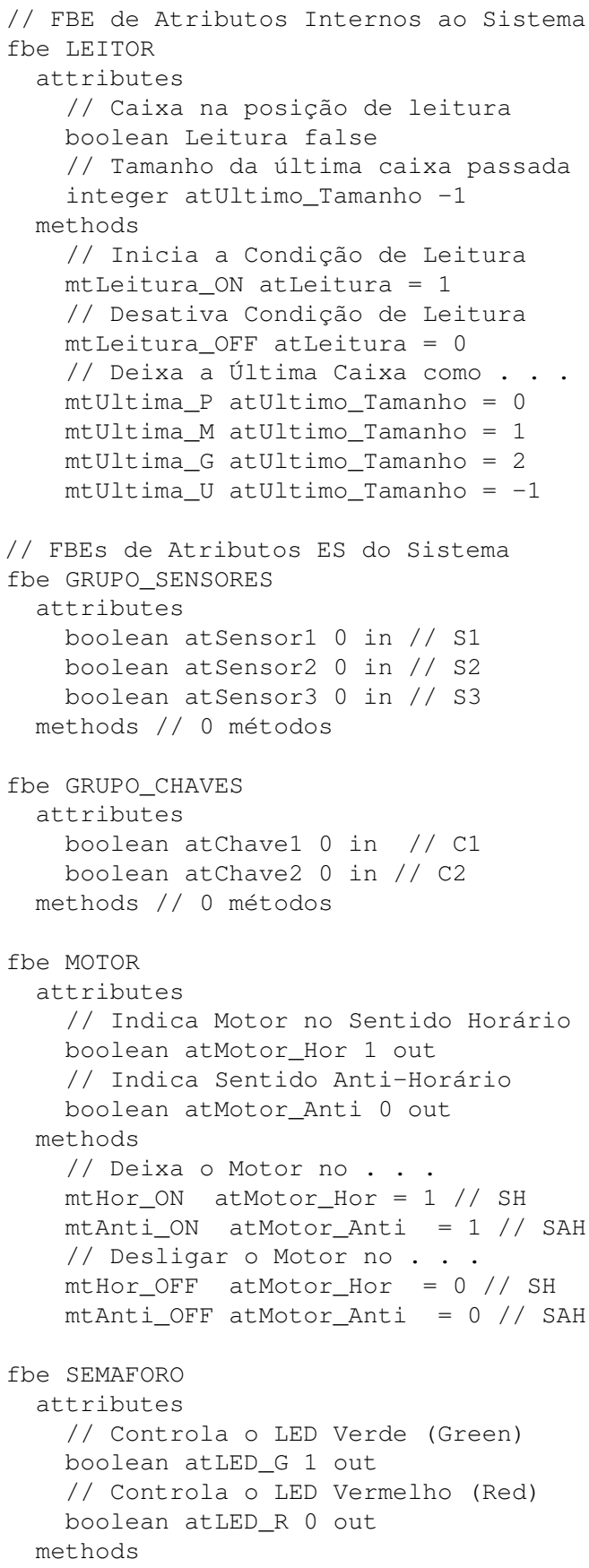




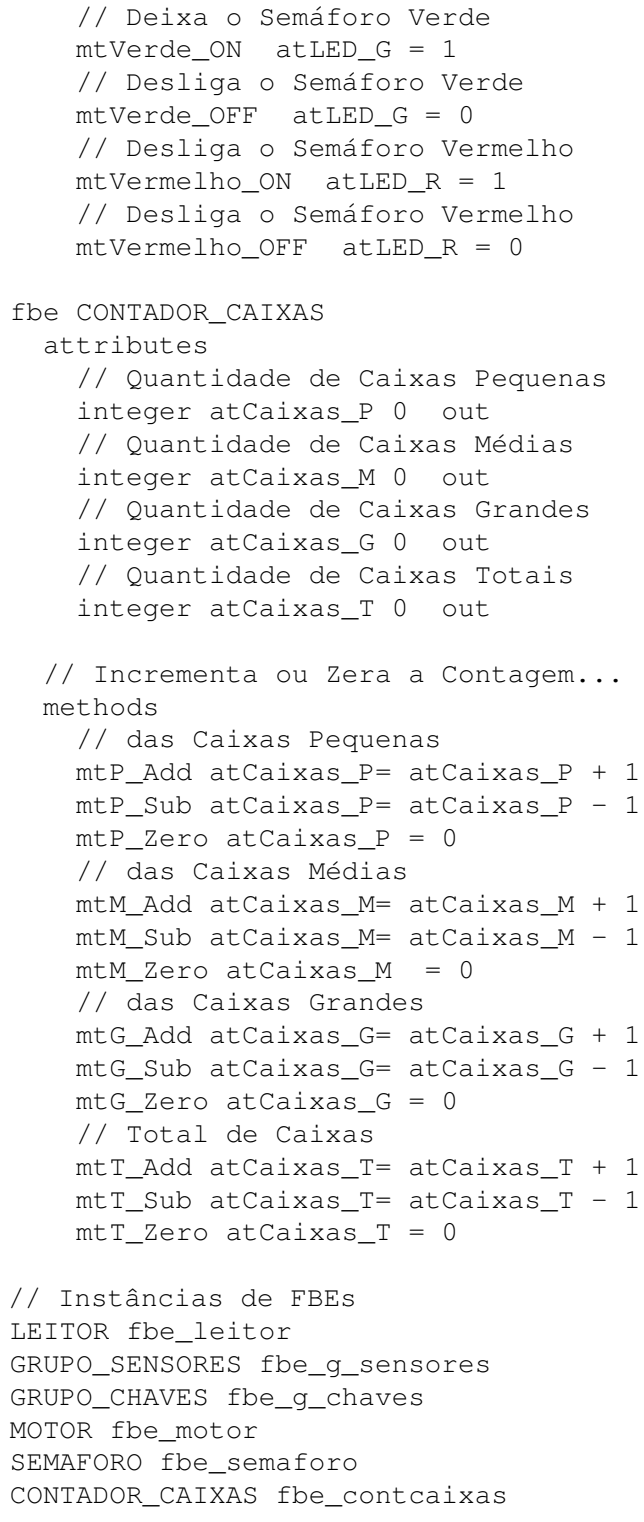

// Instâncias de FBEs

LEITOR fbe leitor

GRUPO_SENSORES fbe_g_sensores

GRUPO_CHAVES fbe_g_chaves

MOTOR fbe_motor

SEMAFORO fbe_semaforo

CONTADOR_CAIXAS fbe_contcaixas

// Criação das Premissas ou Premises

// Verifica se está sendo lido uma caixa premise prLeitura_on fbe_leitor.atLeitur $\mathrm{a}==1$

// Verifica se Não está lendo uma caixa premise prLeitura_OFF fbe_leitor.atLeitu $r a=0$

// Verifica se a Última cx é Incógnita premise prultima_U fbe_leitor.atultimo_T amanho $==-1$

// Verifica se a Última caixa é Pequeno premise prultima_P fbe_leitor.atultimo_T amanho $==0$

// Verifica se a Última caixa é Médio premise prultima_M fbe_leitor.atultimo_T amanho $==1$

// Verifica se a Última caixa é Grande premise prultima_G fbe_leitor.atultimo_T amanho $==2$

// Verifica se Sensor 1 Ativo premise prSensor1_ON fbe_g_sensores.atSe nsor $1==1$
// Verifica se Sensor 1 Não Ativo premise prSensor1_OFF fbe_g_sensores.atS ensor $1=0$

// Verifica se Sensor 2 Ativo premise prSensor2_ON fbe_g_sensores.atSe nsor $2==1$

// Verifica se Sensor 2 Não Ativo premise prSensor2_OFF fbe_g_sensores.atS ensor $2=0$

// Verifica se Sensor 3 Ativo

premise prSensor3_ON fbe_g_sensores.atSe nsor $3==1$

// Verifica se Sensor 3 Não Ativo premise prSensor3_OFF fbe_g_sensores.atS ensor $3==0$

// Verifica Chave C1 está pressionada premise prChave1_ON fbe_g_chaves.atChave $1==1$

// Verifica Chave C1 está solta premise prChave1_OFF fbe_g_chaves.atChav e1 $==0$

// Verifica Chave C2 está pressionada premise prChave2_ON fbe_g_chaves.atChave $2==1$

// Verifica Chave C2 está solta

premise prChave2_OFF fbe_g_chaves.atChav e2 $==0$

// Verifica se O LED Verde está ligado premise prLED_G_ON fbe_semaforo.atLED_G $==1$

// Verifica LED Verde está desligado premise prLED_G_OFF fbe_semaforo.atLED_ $\mathrm{G}==0$

// Verifica LED Vermelho está ligado premise prLED_R_ON fbe_semaforo.atLED_R $==1$

// Verifica LED Vermelho está ligado premise prLED_R_OFF fbe_semaforo.atLED_R $==0$

// Verifica o Limite de Caixas no Pallet premise prexcesso_Caixas fbe_contcaixas. atCaixas_T $==9$

// Verifica se Total de caixas é $>0$ premise prT_Caixas_M_Zero fbe_contcaixas .atCaixas_T > 0

// ---- Conjunto de Regras Relacionadas // ao Excesso de Caixas:

rule rlEC_1 // Liga o Led Vermelho condition prexcesso_Caixas and prLED _G_ON and prSensor1_OFF action instigation fbe_semaforo.mtVe rmelho_ON

rule rleC_2 // Desliga o Led Verde condition prexcesso_Caixas and prLED _G_ON and prSensor1_OFF action instigation fbe_semaforo.mtVe rde_OFF

rule rlEC_3 // Desliga o Motor no SH condition prExcesso_Caixas and prLED _G_ON and prSensor1_OFF action instigation fbe_motor.mtHor_o $\mathrm{FF}$

rule rlEC_4 // Liga o Motor no SAH condition prexcesso_Caixas and prLED _G_ON and prSensor1_OFF action instigation fbe_motor.mtAnti_ ON

rule rlEC_5 // Subtrai 1 do Total 
condition prExcesso_Caixas and prLED _G_ON and prSensor1_OFF

action instigation fbe_contcaixas.mt T_Sub

// Subtrai 1 do P, Se P foi a Última

rule rlEC_6

condition prExcesso_Caixas and prLED _G_ON and prSensor1_OFF and prultima ${ }^{\mathrm{P}}$

action instigation fbe_contcaixas.mt P_Sub

// Subtrai 1 do M, Se M foi a Última

rule rleC_7

condition prExcesso_Caixas and prLED _G_ON and prUltima_M

action instigation fbe_contcaixas.mt M_Sub

// Subtrai 1 do G, Se G foi a Última

rule rleC_8

condition prExcesso_Caixas and prLED _G_ON and prSensor1_OFF and prultima - G

action instigation fbe_contcaixas.mt G_Sub

// ---Conjunto de Regras Relacionadas

// à leitura de uma Caixa

rule rlLE_1 // Ativa Condição de Leitura condition prLeitura_OFF and prLED_G_ ON and prSensor1_ON

action instigation fbe_leitor.mtLeit ura_oN

rule rlLE_2 // Adiciona 1 Ao total de Cx condition prLeitura_OFF and prLED_G_ ON and prSensor1_ON

action instigation fbe_contcaixas.mt T_Add

// Detecta Se é Cx Pequena e soma 1 ao P

rule rlLE_3_1

condition prLeitura_OFF and prLED_G_ $\mathrm{ON}$ and prSensor1_ON and prSensor2_OF $\mathrm{F}$

action instigation fbe_contcaixas.mt P_Add

rule rlLE_3_2 // Complementar à Anterior // Marca a Última Caixa como Pequena condition prLeitura_OFF and prLED_G_ $\mathrm{ON}$ and prSensor1_ON and prSensor2_OF F

action instigation fbe_leitor.mtUlti ma_P

// Detecta Se é Cx Média e soma 1 ao M

rule rlLE_4_1

condition prLeitura_OFF and prLED_G_ ON and prSensor1_ON and prSensor3_OF F

action instigation fbe_contcaixas.mt M_Add

rule rlLE_4_2 // Complementar à Anterior // Marca a Última Caixa como Média condition prLeitura_OFF and prLED_G_ ON and prSensor2_ON and prSensor3_OF F

action instigation fbe_leitor.mtUlti ma_M

// Detecta Cx Grande e Adiciona 1 ao G

rule rlLE_5_1

condition prLeitura_OFF and prLED_G_ ON and prSensor1_ON and prSensor2_ON and prSensor3_ON action instigation fbe_contcaixas.mt G_Add

rule rlLE_5_2 // Complementar à Anterior // Marca a Última Caixa como Grande condition prLeitura_OFF and prLED_G_ ON and prSensor1_ON and prSensor2_ON and prSensor3_ON

action instigation fbe_leitor.mtulti ma_G

// Se estava em Condição de Leitura, //quando a caixa passa

rule rlLE_6

// do Sensor 1, acabou a leitura condition prLeitura_ON and prLED_G_O $\mathrm{N}$ and prSensor1_OFF action instigation fbe_leitor.mtLeit ura_OFF

// Conj. Regras Relacionadas a pressionar a Chave 1 (Parar Operação)

rule rlC1_1 // Desliga o LED Verde condition prChave1_ON and prLED_G_ON action instigation fbe_semaforo.mtVe rde_OFF

rule rlC1_21 // Liga o LED Vermelho condition prChave1_ON and prLED_G_ON action instigation fbe_semaforo.mtVe rmelho_ON

rule rlC1_3 // Se Lendo Desativa Leitura condition prChave1_ON and prLED_G_ON action instigation fbe_leitor.mtLeit ura_OFF

rule rlC1_4 // Subtrai 1 Caixa do Total condition prChave1_ON and prLED_G_ON and prT_Caixas_M_zero

action instigation fbe_contcaixas.mt T_Sub

rule rlC1_5_1 // Subtrai 1 Caixa de P, //Se última Caixa Pequena condition prChave1_ON and prLED_G_ON and prultima_P

action instigation fbe_contcaixas.mt P_Sub

rule rlC1_5_2 // Subtrai 1 Caixa de M, //Se última Caixa Média

condition prChave1_ON and prLED_G_ON and prultima_M

action instigation fbe_contcaixas.mt M_Sub

rule rlC1_5_3 // Subtrai 1 Caixa de G, //Se última Caixa Grande condition prChave1_ON and prLED_G_ON and prultima_G action instigation fbe_contcaixas.mt G_Sub

rule rlC1_6 // Desliga o Motor do SH condition prChave1_ON and prLED_G_ON action instigation fbe_motor.mtHor_O $\mathrm{FF}$

rule rlC1_7 // Liga o Motor no SAH condition prChave1_ON and prLED_G_ON action instigation fbe_motor.mtAnti_ ON

// Conj. Regras Relacionadas pressionar // Chave 2 Retomar Operação

rule rlC2_1 // Liga o LED Verde condition prChave2_ON and prLED_R_ON action instigation fbe_semaforo.mtVe rde_ON 
rule rlc2 2 // Desliga o LED Vermelho condition prChave2_ON and prLED_R_ON action instigation fbe_semaforo.mtVe rmelho_OFF

rule rlC2_3 // Zera o Contador Cx Total condition prChave2_ON and prLED_R_ON action instigation fbe_contcaixas.mt T_zero

rule rlC2_4 // Zera Contador Cx Pequenas condition prChave2_ON and prLED_R_ON action instigation fbe_contcaixas.mt P_Zero

rule rlC2_5 // Zera o Contador Cx Médias condition prChave2_ON and prLED_R_ON action instigation fbe_contcaixas.mt M_Zero

rule rlC2_6 // Zera Contador Cx Grandes condition prChave2_ON and prLED_R_ON action instigation fbe_contcaixas.mt G_Zero

rule rlC2_7 // Desliga Motor no SAH condition prChave2_ON and prLED_R_ON action instigation fbe_motor.mtAnti_ $\mathrm{OFF}$

rule rlc2_8 // // Liga o Motor no SH condition prChave2_ON and prLED_R_ON action instigation fbe_motor.mtHor_o $\mathrm{N}$

rule rlc2_9 // Última caixa Incógnita condition prChave2_ON and prLED_R_ON action instigation fbe_leitor.mtUlti ma_U

// ---Conjunto de Regras Inicialização

// (Retomar Operação)

rule rlintI_1

// Garante início - Led verde - ON condition prLED_G_OFF and prLED_R_OF F

action instigation fbe_semaforo.mtVe rde_ON

Por fim, este código todo pode ser encontrado em formato original, no penúltimo anexo da tese de doutorado do $1^{\circ}$ autor, em LingPON-HD dito prototipal [9]. Nesta versão prototipal, as palavras chaves dos FBEs e afins são suprimidos, sendo os Attributes e os Methods tidos como globais. Isto era considerado aceitável dado que os FBEs não geram hardware, mas sim seus Attributes e seus Methods [9].

Ainda, conforme já descrito, no experimento alguns outros elementos de hardware foram utilizados, como display de sete segmentos, chaves de duas posições e alguns $L E D$ s. Quanto as chaves de duas posições, verificou-se que um ruído é gerado da sua comutação. Assim, foi necessário o tratamento deste sinal.

Para tanto, foi elaborada uma lógica por meio da tecnologia LingPON-HD para o controle de debouncing. Foram utilizados para tal 12 Premises, 14 Rules e FBEs com 6 Attributes e 9 Methods. A seguir é apresentado este programa em linguagem declarativa da tecnologia LingPON-HD.

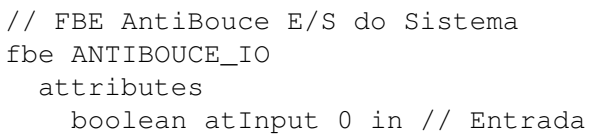

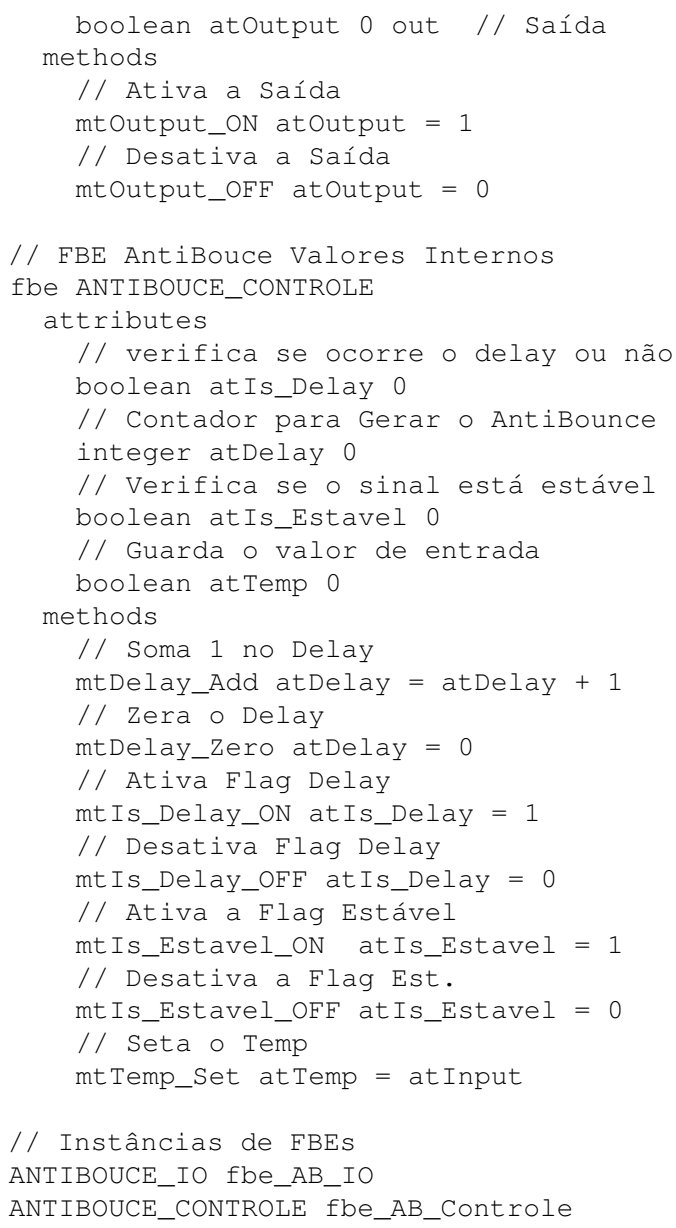




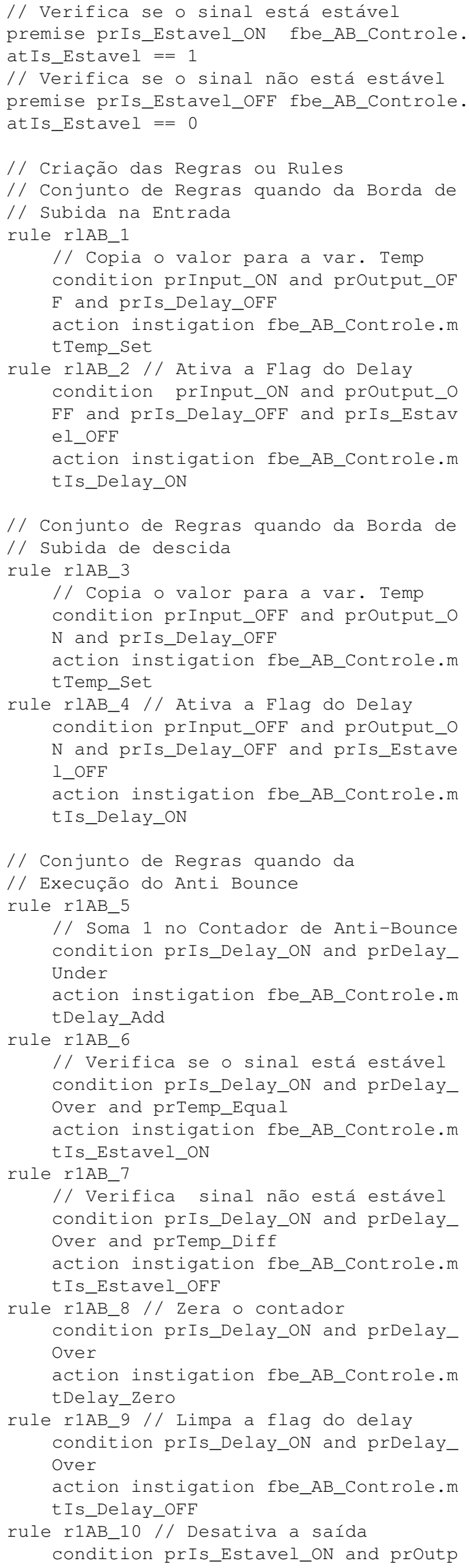

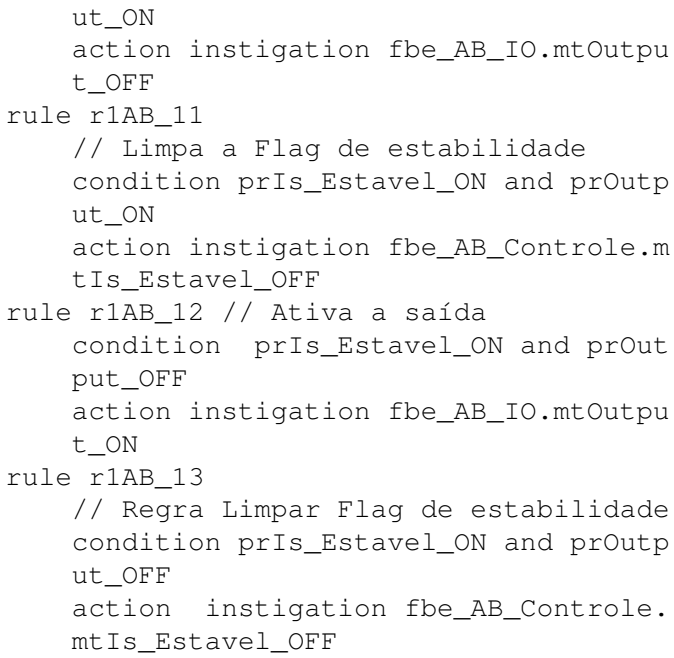

Os circuitos resultantes da compilação em VHDL dos módulos feitos em tecnologia LingPON-HD foram integrados com o restante do sistema. Todo o sistema foi gravado na FPGA por meio de um computador utilizando o software Quartus II.

\subsection{Resultados Obtidos}

Por meio da solução elaborada e criada via Tecnologia Ling PON-HD, combinando os módulos resultantes compostos das entidades PON (monitor e debounce) com os demais módulos do sistema, foi construído um projeto totalmente funcional que atendia os requisitos do problema apresentado no tocante a monitoração de quantidades de caixas de papelão. $\mathrm{O}$ sistema criado funcionou perfeitamente e sem falhar, sendo o experimento de todo funcional.

$\mathrm{O}$ experimento tratado neste artigo permite convalidar o funcionamento da Tecnologia LingPON-HD como linguagem e ferramenta de desenvolvimento em alto-nível de códigos para sintetização de Hardware Digital especializado. A diferença em relações aos diversos experimentos prévios e mesmo subsequentes feitos em PON-HD é que este se deu por equipe de desenvolvimento independente e não experimentata que aprendeu a Tecnologia LingPON e a usou em alto nível sem intervenções técnicas maiores.

Tal qual nos demais experimentos, com esse experimento realizado também foi possível observar que a construção de entidades se utilizando da Tecnologia LingPON-HD é suficientemente flexível e pode ser modularizada, de forma a possibilitar que os problemas sejam subdivididos e resolvidos de forma independente. Isto ocorreu no caso quando foi optado pelo desenvolvimento de módulo individual para tratamento do debouce, além do módulo de monitoração

Estes módulos independentes são fundamentais uma vez que podem ser facilmente conectados com outros módulos por meio de suas portas de entrada e saída, em termo de circuito. Isto já se mostra uma qualidade no tocante a facilidades de desacoplamento entre os módulos, portanto havendo potencial para uso da ferramenta em projetos de maior complexidade. 
Ademais, por meio deste trabalho foi possível a validação da linguagem em si e da documentação como ferramenta de ensino dos conceitos do PON, uma vez que os desenvolvedores que fizeram os dois módulos usando a Tecnologia LingPON-HD são discentes de graduação que não haviam tido contato anterior com o PON. Por meio da documentação existente e de alguns exemplos, eles foram capazes de se utilizar desta tecnologia e desenvolver a aplicação sem a influência de outrem. Assim, observa-se o potencial da ferramenta em si, bem como sua contribuição para o desenvolvimento do próprio PON.

\section{Conclusão}

O PON vem apresentando resultados positivos em várias plataformas, conforme revisou este presente trabalho. No entanto, por hora a única implementação que reproduz com precisão o modelo conceitual paralelo do PON é o PON-HD. De fato, a implementação do PON em hardware digital permite que as notificações aconteçam em paralelo, maximizando o desempenho do sistema, conforme demonstra o estado da arte em PON-HD também revisado neste artigo.

Com o experimento realizado neste trabalho, concordando com o estado da arte em PON-HD, foi possível validar a facilidade de criação dos circuitos via Tecnologia LingPONHD. No caso do experimento apresentado, ele foi feito por dois desenvolvedores que pouco conheciam do PON e nada da arquitetura PON-HD, tendo como base apenas conhecimentos prévios de programação já adquiridos em outros paradigmas computacionais. Em verdade, bastou uma simples apresentação dos conceitos e a utilização de manuais com instruções sucintas sobre a LingPON-HD para que eles fossem capazes de desenvolver uma aplicação funcional, que apresenta interfaces com o sistema simulado dado.

Neste contexto, o presente artigo enfatiza a validade da Tecnologia LingPON-HD como uma potencial ferramenta de alto nível para o desenvolvimento de hardware. De fato, essa Tecnologia LingPON-HD é um caminho para permitir a síntese de hardware sem necessitar conhecimento de linguagens mais complexas como VHDL ou Verilog. Ademais, isto se dá ainda com garantia de paralelismo implícito e, quando comparado com abordagens tradicionais, sem qualquer perda relevante na performance, área ou tamanho de circuito e frequência máxima de operação, cf. estudos prévios realizados em PON-HD [4][9][21][22].

Em suma, como a Tecnologia LingPON-HD faz uso de uma linguagem declarativa simples e em alto nível, ademais empregando componentes previamente testados, ficaria mais fácil criar os circuitos e a possibilidade de erros é reduzida. Ainda, o paralelismo propiciado pela natureza do PON contribui para uma boa performance dos circuitos gerados em VHDL via PON-HD. Assim, no tocante as contribuições deste artigo, pode-se concluir que a Tecnologia LingPON-HD poderia ser evoluída e ainda mais investigada, inclusive como uma ferramenta de síntese de alto nível.

\section{Agradecimentos}

Os autores agradecem os esforços do grupo de pesquisa do PON, em particular aos Prof. P. C. Stadzisz, Prof. J. A. Fabro, Prof. R. R. Linhares, Dr. A. F. Ronszcka e M. Sc. L. F. Pordeus, tal qual a definição pelo Prof. H. S. Lopes do problema considerado, o que inspirou parte da descrição usada neste presente artigo, bem como a confecção da Figura 1.

\section{Contribuição dos autores}

- Ricardo Kerschbaumer é o desenvolvedor do Paradigma Orientado a Notificações para Hardware Digital, (PONHD), foi responsável pela redação do artigo, pela compilação das informações e pela busca das referências bibliográficas.

- Os autores André Augusto Kaviatkovski e Gabriel Rodrigues Garcia foram responsáveis pela implementação descrita no artigo, desenvolveram os códigos e realizaram os testes. Também foram revisores do artigo, contribuindo principalmente com a parte da implementação.

- O autor Carlos Raimundo Erig Lima foi o professor responsável por propor o experimento e supervisionar a implementação dos códigos e os testes realizados, no artigo contribuiu com parte do texto que descreve a implementação bem como na revisão do artigo como um todo.

- O autor Jean Marcelo Simão é o proponente do Paradigma Orientado a Notificações, (PON), tendo contribuído com a redação do artigo como um todo e nas revisões bibliográficas.

- Foi também contribuição de Jean Marcelo Simão, junto com Ricardo Kerschbaumer, a redação das respostas aos revisores durante o processo de submissão do artigo.

\section{Referências}

[1] CROSBIE, R. Using field-programmable gate arrays for high-speed real-time simulation. International Journal of Modeling, Simulation, and Scientific Computing, v. 1, n. 1, p. 99-115, 2010. Disponível em: 〈https://www.worldscientific. com/doi/abs/10.1142/S1793962310000031 $\rangle$.

[2] QIAN, J. et al. Fpga acceleration window joins over multiple data streams. International Journal of Modeling, Simulation, and Scientific Computing, v. 14, n. 4, p. 813-830, 2005. Disponível em: 〈https://www.worldscientific.com/doi/ abs/10.1142/S0218126605002611 $\rangle$.

[3] BORKAR, S.; CHIEN, A. A. The future of microprocessors. Communications of the ACM, v. 54, n. 5, p. 67-77, 2011. Disponível em: 〈https: //dl.acm.org/doi/10.1145/1941487.1941507〉. 
[4] KERSCHBAUMER, R. et al. Notification oriented paradigm to implement digital hardware. Journal of Circuits Systems and Computers, v. 27, n. 8, 2018. Disponível em: 〈https://www.worldscientific.com/doi/10.1142/ S0218126618501244〉.

[5] RONSZCKA, A. F. et al. Notification-oriented paradigm framework 2.0: An implementation based on design patterns. IEEE Latin America Transactions, v. 15, n. 11, 2017. Disponível em: $\langle$ https://ieeexplore.ieee.org/document/8070430〉.

[6] RONSZCKA, A. F. et al. Notification-oriented and rete network inference: A comparative study. In: IEEE International Conference On Systems, Man and Cybernetics Conference. Kowloon, Hong-Kong, China: IEEE, 2015. Disponível em: $\langle$ https://ieeexplore.ieee.org/document/7379282〉.

[7] RONSZCKA, A. F. et al. Notification oriented programming language and compiler. In: VII Brazilian Symposium on Computing Systems Engineering - SBESC. Curitiba, Paraná, Brazil: IEEE Xplore, 2017.

[8] LOPES, H. S. Exercício da esteira - laboratório 1. disciplina sistema controlados," disciplina: Sistemas microcontrolados (códigos: Eex22-s71 - if64j-s71). cursos: Engenharia eletrônica e engenharia da computação. departamento acadêmico de eletrônica (daeln). In: . [s.n.], 2017. Disponível em: 〈https://pessoal.dainf.ct.utfpr.edu.br/ jeansimao/PON/lab1-1-2017.pdf $\rangle$.

[9] KERSCHBAUMER, R. Proposição do paradigma orientado a notificações no desenvolvimento de circuitos lógico-digitais reconfiguráveis. $378 \mathrm{p}$. Tese (Doutorado) — Programa de Pós-graduação em Engenharia Elétrica e Informática Industrial (CPGEI) - Universidade Tecnológica Federal do Paraná (UTFPR), Curitiba, Paraná, Brasil, 2018. Disponível em: $\langle$ http://repositorio.utfpr.edu.br/jspui/handle/1/3811〉.

[10] SIMãO, J. M.; STADZISZ, P. C. Paradigma Orientado a Notificações (PON) - Uma Técnica de Composição e Execução de Software Orientado a Notificações. 2008. Pedido de Patente. UTFPR (Agência de Inovação 2007), INPI N. PI08055181. Disponível em: 〈https://pessoal.dainf.ct.utfpr. edu.br/jeansimao/PON/PON_Patentes.htm $\rangle$.

[11] SIMãO, J. M.; STADZISZ, P. C. Inference based on notifications: a holonic metamodel applied to control issues. IEEE Transactions on Systems, Man, and Cybernetics - Part A: Systems and Humans, v. 39, n. 1, p. 238-250, 2009. Disponível em: $\langle$ https://ieeexplore.ieee.org/document/4689369〉.

[12] LINHARES, R. R. et al. Evaluation of the notification oriented paradigm applied to real-time systems. In: $I V$ Brazilian Symposium on Computing Systems Engineering - SBESC. Manaus, Amazonas, Brazil: IEEE Xplore, 2014. Disponível em: 〈https://sbesc.lisha.ufsc.br/sbesc2014/Anais〉.
[13] SIMãO, J. M. et al. Evaluation of the notification oriented paradigm applied to sentient computing. In: 10th Workshop on Software Technologies for Future Embedded and Ubiquitous Systems (SEUS 2014) / IEEE 17th International Symposium on Object/Component/ServiceOriented Real-Time Distributed Computing (ISORC 2014). Reno, Nevada, USA: IEEE, 2014. Disponível em: 〈https://ieeexplore.ieee.org/abstract/document/6899157〉.

[14] OLIVEIRA, R. N. et al. Notification oriented paradigm applied to ambient assisted living tool. IEEE Latin America Transactions, v. 16, n. 2, p. 647 -653, 2018. Disponível em: $\langle$ https://ieeexplore.ieee.org/document/8327425〉.

[15] SIMãO, J. M. et al. Paradigma Orientado a Notificações em Hardware Digital. 2012. Pedido de Patente. - UTFPR (Agência de Inovação 11 de novembro de 2012), INPI N. BR 102012026429 3. Disponível em: 〈https://pessoal.dainf.ct. utfpr.edu.br/jeansimao/PON/PON_Patentes.htm $\rangle$.

[16] RONSZCKA, A. F. Método para a Criação de Linguagens de Programação e Compiladores para o Paradigma Orientado a Notificações em Plataformas Distintas. Tese (Doutorado) — Programa de Pós-graduação em Engenharia Elétrica e Informática Industrial (CPGEI) - Universidade Tecnológica Federal do Paraná (UTFPR), Curitiba, Paraná, Brasil, 2019. Disponível em: $\langle$ http://repositorio.utfpr.edu.br/jspui/handle/1/4234〉.

[17] PETERS, E. et al. A new hardware coprocessor for accelerating notification-oriented applications. In: 2012 International Conference on Field-Programmable Technology (FTP). Seoul, South Korea: [s.n.], 2012. p. 10-12.

[18] LINHARES, R. R.; SIMãO, J. M.; STADZISZ, P. C. Noca - a notification-oriented computer architecture. IEEE Latin America Transactions, v. 13, n. 5, p. 1593-1604, 2015. Disponível em: $\langle$ https://ieeexplore.ieee.org/document/7112020 .

[19] LINHARES, R. et al. Noca - a notification-oriented computer architecture: Prototype and simulator. IEEE Access, v. 8, p. 37287-37304, 2020. Disponível em: $\langle$ https://ieeexplore.ieee.org/document/9004603〉.

[20] PORDEUS, L. F. et al. Notification oriented paradigm to digital hardware. Revista SODEBRAS, v. 11, n. 128, 2016. Disponível em: 〈http://www.sodebras.com.br/edicoes/N128. pdf $\rangle$.

[21] KERSCHBAUMER, R. et al. A tool for digital circuits synthesis based on notification oriented paradigm. IEEE Latin America Transactions, v. 16, n. 6, p. 1574-1586, 2018. Disponível em: $\langle$ https://ieeexplore.ieee.org/document/8444152〉.

[22] PORDEUS, L. F. et al. Nop-dh - evaluation over bitonic sort algorithm,. Microprocessors and Microsystems, v. 85, p. 104314, 2021. Disponível em: 〈https://www.sciencedirect. com/science/article/abs/pii/S0141933121004750 $\rangle$.

[23] SCHüTZ, F. NeuroPON: Uma Abordagem para

o Desenvolvimento de Redes Neurais Artificiais 
utilizando o Paradigma Orientado a Notificações. Tese (Doutorado) - Programa de Pós-graduação em Engenharia Elétrica e Informática Industrial (CPGEI) - Universidade Tecnológica Federal do Paraná (UTFPR), Curitiba, Paraná, Brasil, 2019. Disponível em: $\langle$ http://repositorio.utfpr.edu.br/jspui/handle/1/4487〉.

[24] SCHüTZ, F. et al. Proposal of a declarative and parallelizable artificial neural network using the notification-oriented paradigm. Neural Computing and Applications, Springer, v. 4, n. 30, p. 1715-1731, 2018. Disponível em: $\langle$ https://rdcu.be/S5DJ $\rangle$.

[25] TERASIC. Altera DE1 Board. Acessado dia 30 de julho de 2021. Disponível em: 〈https: //www.terasic.com.tw/cgi-bin/page/archive.pl?Language= English $\&$ Category $\mathrm{No}=183 \& \mathrm{No}=83 \& \mathrm{PartNo}=3\rangle$. 